\title{
Optimal Growth of Linear Perturbations in Low Pressure Turbine Flows
}

\author{
Atul S Sharma ${ }^{1}$, Nadir Abdessemed ${ }^{1}$, Spencer Sherwin ${ }^{1}$, Vassilis Theofilis ${ }^{2}$ \\ 1 Imperial College, London, SW7 2BT, UK \\ 2 School of Aeronautics, Universidad Politécnica de Madrid, Pza. Cardenal Cisneros \\ 3, E-28040 Madrid, Spain
}

\begin{abstract}
This paper presents a numerical algorithm for the linearized flow problem involving complex geometries where analytical solution is impossible. The method centres around calculation of an eigenvalue problem involving the linearised flow and its spatial adjoint, and yields the flow perturbations that grow the most in a prescribed time, the magnitude of that growth and the perturbations after the growth has occurred. Previous work has shown that classical stability analysis of flow past a low-pressure turbine blade gives only stable eigenvalues, which cannot explain transition to turbulence in this flow. The inital value problem for this fan blade is presented and demonstrates significant perturbation growth, indicating that this growth may be the facilitator for transition in this case.
\end{abstract}

\section{Introduction}

The mechanisms of transition from two-dimensional to three-dimensional flow have been extensively investigated for canonical problems such as flat-plate boundary-layer and channel flows and for problems involving more complex geometries, such as the flow past a circular cylinder. For the latter case linear stability analysis has been successfully applied to identify the growing eigenmodes [1] responsible for transition and the onset to turbulence.

The analysis typically proceeds by considering the growth of small perturbations on a known, laminar solution to the Navier-Stokes equations. A subsequent eigenmode analysis yields a Reynolds number above which an instability occurs. In plane Poiseuille flow for instance Tollmien-Schlichting waves grow exponentially (in the linear regime) above that critical Reynolds number and transition occurs via a secondary instability resulting from non-linear effects.

In a recent study [2] the flow past a low-pressure turbine blade (LPT) has been investigated in order to understand the instability mechanisms in this class of flows. This understanding might be crucial for controlling the laminar boundary layer separation which has experimentally shown to increase turbine performance. The initial study showed that eigenmodes obtained using classical linear stability analysis are all stable for the entire investigated parameter space comprising the Reynolds number Re and the spanwise perturbation wave lengths 
$L_{z}$. This means that modal analysis with secondary instabilities cannot explain transition in this flow.

This apparent paradox can be understood mathematically in terms of system non-normality. Even when the system linearised about a steady flow is asymptotically stable, it can exhibit large transient growth of the perturbation energy before returning to equilibrium. Physically, this growth is understood to be fed by the transport of energy from the steady flow to the perturbations. Perturbations may then grow in accordance with the linear model at sub-critical Reynolds number, to become large enough for nonlinearities to become significant. Transition then occurs via secondary instability, 'bypassing' the classical mechanism [3]. In flows such as plane Poiseuille flow, this 'transient growth' has been shown to explain transition [4-6] and previous studies [7] suggest similarities to the flow past the LPT blade. The present study aims to identify modes that are associated to this optimum growth of perturbations as a result of this non-normality.

The initial condition with the most energy growth at time $t$ was found by a calculus of variations method in [4], for Couette flow and for Poiseuille flow. An approach based on the singular value decomposition of the mapping from the initial condition $x_{0}$ to the state $x(t)$ at time $t$ is found in [6]. The current work extends that theory by presenting an algorithm allowing calculation of this decomposition for complex geometry flows where an analytical treatment is prohibitively difficult if not impossible.

\section{Method}

The Schmidt decomposition of $B(t)$ in the linear evolution equation $x(t)=$ $B(t) x_{0}$ can be expressed in terms of an eigenvalue problem involving $B(t)$ and its adjoint $B^{*}(t)$. Specifically, the singular values of $B(t)$ are the square roots of the eigenvalues of $B^{*}(t) B(t)$ and the right Schmidt vectors of $B(t)$ are the eigenvectors of $B^{*}(t) B(t)$. Where $B(t)$ and its adjoint are evaluated by numerical simulation, this eigenvalue problem can be solved numerically, allowing identification of the Schmidt pairs associated with the largest singular values. The first right Schmidt vector of $B(t)$ gives the initial condition associated with growth of magnitude of the first singular value. The first left Schmidt vector is the final condition that the initial condition evolves into. In this way, the optimal subspace can be found for complex problems where the operators are not known (or determinable) analytically. These Schmidt pairs are associated with the 'optimals' discussed in [4].

The eigenvalues of the compound operator $B^{*}(t) B(t)$ can be computed using an Arnoldi algorithm. The action of $B$ can be expressed by integrating the linearised Navier-Stokes equations via the linear time stepping scheme of [8]

$$
x(t+\Delta t)=(\mathbf{I}-\Delta t \mathbf{L})^{-1}\left(\mathbf{I}+\Delta t \mathbf{N}_{\mathbf{U}}\right) x(t),
$$

whose computational implementation is based on a time splitting scheme [9] and discretised using a spectral $/ h p$ element technique to capture the flow solution 
in the complex geometry. Similarly the action of $B^{*}(t)$ can be expressed by integrating over its spatial adjoint system. Time marching those systems allows the computation of the eigenvalues of $B^{*}(t) B(t)$ to yield the singular values of $B(t)$.

\section{Optimal Growth Modes in Low Pressure Turbine Flow}

The following results are concerned with the optimal two-dimensional perturbations for the steady base flow past the Low Pressure Turbine blade, whose linear stability has been extensively investigated in previous studies $[2,10]$. Considering the complex two-dimensional configuration of the blade shows the mode associated to the largest singular value. A strong energy concentration can be made out in the shear layers around the separation bubble (Fig. 1), which is suspected to be the origin of instability and transient growth for this flow. The modes identified in a preceeding pseudo-spectrum analysis [10] substantiates the potential for growth within the bubble, which raises the question of receptivity of the here presented mode vs. sensitivity due to forcing of the latter one Considering how the perturbation evolves in a given time, one can seen that the

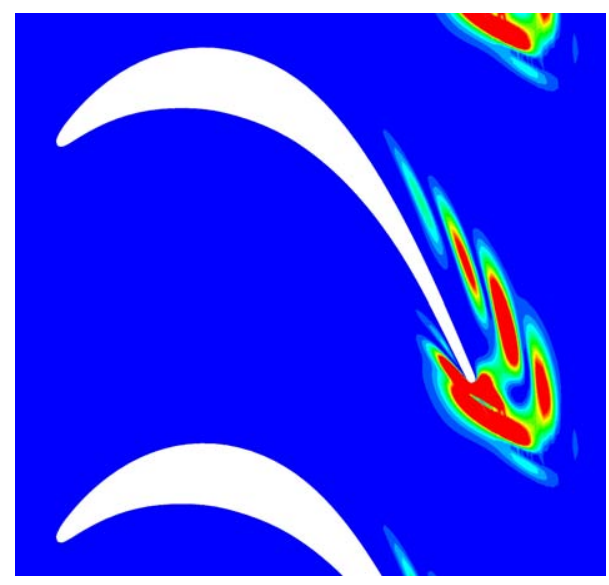

Fig. 1. Initial condition singular vector (Eigenmode of $B^{*} B$ ), $\sigma_{1}=418$. The high value indicates that non-normality is important in this case.

energy of the here identified mode grows significantly as it travels downstream (Fig. 2).

\section{Acknowledegments}

A Sharma is funded by EPSRC, BAE systems through the FLAVIIR project and N. Abdessemed is supported through AFSOR in collaboration with Professor V. 


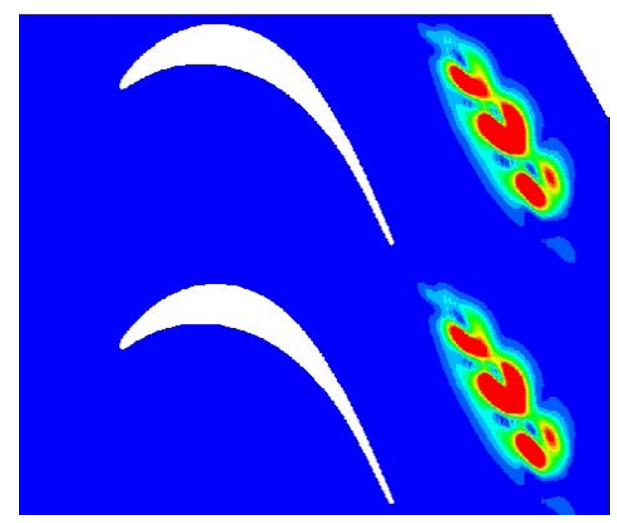

Fig. 2. Final condition SVD-mode (Eigenmode of $B B^{*}$ ).

Theofilis at the Technical University of Madrid. The authors would also like to acknowledge insightful discussion with Professor Dwight Barkley of Warwick University and Professor David Limebeer at Imperial College London.

\section{References}

1. Barkley, D., Henderson, R.: Three-dimensional floquet stability analysis of the wake of a circular cylinder. 322 (1996) 215 - 241

2. Abdessemed, N., Sherwin, S., Theofilis, V.: On unstable 2d basic states in low pressure turbine flows at moderate reynolds numbers, AIAA Paper 2004-2541 (2004)

3. Reddy, S., Schmid, P.J., Baggett, J.S., Henningson, D.S.: On stability of streamwise streaks and transition thresholds in plane channel flows. J. Fluid Mech. 365 (1998) 269-303

4. Butler, K., Farrell, B.: Three-dimensional optimal perturbations in viscous shear flow. Phys. Fluids 4(8) (1992)

5. Trefethen, L.N., Trefethen, A.E., Reddy, S., Driscoll, T.A.: Hydrodynamic stability without eigenvalues. Science 261(5121) (1993)

6. Schmid, P.J., Henningson, D.S.: Stability and Transistion in Shear Flows. SpringerVerlag, New York (2001)

7. Wu, X., Durbin, P.A.: Evidence of longitudinal vortices evolved from distorted wakes in a turbine passage. 446 (2001) 199-228

8. Tuckerman, L., Barkley, D.: Bifurcation analysis for timesteppers. In Doedel, E., Tuckerman, L., eds.: Numerical Methods for Bifurcation Problems and Large-Scale Dynamical Systems. Volume 119., Springer, New York (2000) 543-466

9. G. Karniadakis, M.I., S.Orszag: High-order splitting methods for the incompressible navier stokes equations. Journal of computational Physics 97 (1991) 414-443

10. Abdessemed, N., Sherwin, S., Theofilis, V.: Linear stability of the flow past a low pressure turbine blade, AIAA Paper 2006-3530 (2006) 